https://helda.helsinki.fi

\title{
Insomnia symptoms and short sleep duration predict trajectory of mental health symptoms
}

\section{Biddle, Daniel J.}

2019-02

Biddle , D J , Hermens , D F , Lallukka , T , Aji , M \& Glozier , N 2019 , ' Insomnia symptoms and short sleep duration predict trajectory of mental health symptoms ' , Sleep Medicine , vol. 54 , pp. 53-61 . https://doi.org/10.1016/j.sleep.2018.10.008

http://hdl.handle.net/10138/310986

https://doi.org/10.1016/j.sleep.2018.10.008

cc_by_nc_nd

acceptedVersion

Downloaded from Helda, University of Helsinki institutional repository.

This is an electronic reprint of the original article.

This reprint may differ from the original in pagination and typographic detail.

Please cite the original version. 
2 Title: Insomnia symptoms and short sleep duration predict trajectory of mental health

3

4

\section{Author Names \& Affiliations:}

Daniel J. Biddle ${ }^{\mathrm{a}}$, Daniel F. Hermens ${ }^{\mathrm{a}, \mathrm{b}}$, Tea Lallukka ${ }^{\mathrm{a}, \mathrm{c}, \mathrm{d}}$, Melissa Aji ${ }^{\mathrm{a}}$, Nick Glozier ${ }^{\mathrm{a}}$

${ }^{a}$ Brain and Mind Centre, School of Medicine and Health, University of Sydney, 94 Mallett St, Camperdown, NSW 2050, Australia

${ }^{\mathrm{b}}$ Sunshine Coast Mind and Neuroscience - Thompson Institute (ML59), University of the Sunshine Coast, Locked Bag 4, Maroochydore DC QLD 4558, Australia

${ }^{c}$ Finnish Institute of Occupational Health, PO Box 18 FI-00032, Työterveyslaitos, Finland

${ }^{\mathrm{d}}$ Department of Public Health, PO Box 20 (Tukholmankatu 8 B) 00014, University of Helsinki, Finland

Corresponding author: Nick Glozier, $\mathrm{PhD}$

Mailing address: Professor Marie Bashir Centre, Level 5, Building 11 RPAH, 67-73

Missenden Road, Camperdown, NSW 2050, Australia

Phone: +61 295151596

Fax: +61 293510855

Email: nick.glozier@sydney.edu.au 


\section{$1 \quad$ Abstract \& Keywords}

3 Objectives: Evaluate whether insomnia symptoms and short or long sleep duration, alone or

4 in combination, are robustly associated with subsequent trajectory of mental health

5 symptoms.

6

7 Methods: Participants were 2,598 individuals (15-to-94 years of age) with elevated mental

8 health symptoms at baseline (2013-14). Associations of baseline insomnia symptoms and

9 sleep duration with two-year trajectory of mental health were estimated and adjusted for

10 multiple potential confounders. Outcomes included recovery (well at both follow-up

11 timepoints), intermittent symptoms (unwell at one follow-up timepoint), and chronic symptoms (unwell at each follow-up timepoint).

14 Results: Adjusted for age and sex, baseline insomnia symptoms predicted intermittent (OR $1.43,95 \%$ CI 1.15-1.80) and chronic (OR 2.16, 95\% CI 1.77-2.68) trajectories of mental health symptoms. Short sleep duration ( $<6 \mathrm{~h}$ and $\geq 6$ to $<7 \mathrm{~h})$ only predicted a chronic trajectory (ORs 1.70-2.06). Associations were attenuated but significant after confounder adjustment. Those who experienced both insomnia and short $(<7 \mathrm{~h})$ sleep duration had the greatest risk of chronic mental health symptoms (OR 2.35, 95\% CI 1.60-3.45).

21 Conclusion: A focus on just sleep duration or insomnia symptoms in those with elevated mental health symptoms will not be adequate to address chronicity. Both components of sleep disturbance, and in particular their co-occurrence, should be addressed.

Keywords: Insomnia; Sleep Duration; Prognosis; Depression; Anxiety 


\section{$1 \quad$ Main Text}

2 Insomnia symptoms and short sleep duration predict trajectory of mental health

3 symptoms.

\section{1. Introduction}

5 There is a bi-directional link between sleep disturbance and mental health symptoms, with

6 disordered sleep representing both a symptom of and risk factor for the development of

7 common mental disorders. ${ }^{1-6}$

8 Sleep disturbance is a multidimensional concept, comprised of such phenomena as insomnia

9 symptoms, sleep duration, and sleep schedule/timing. ${ }^{7}$ Recent research has explored how

10 these sleep disturbance dimensions combine to predict mental health incidence and

11 prevalence, ${ }^{7-11}$ with a particular focus on the combination of insomnia symptoms and short

12 sleep duration.

13 One large-scale cross-sectional study $(n=3911)$ found that those experiencing insomnia with

14 short sleep duration display higher risk of depression than those with insomnia but normal

15 sleep duration, and greater prevalence of depression and anxiety than healthy-sleeping

16 controls. ${ }^{8}$ In another large-scale study $(n=1137)$, Fernandez-Mendoza et al. found that of the

17 sleep subgroups assessed, the odds of incident depression at 7.5 year follow-up were highest

18 among those with insomnia and short sleep duration, and that this association was

19 independent of psychological coping resources. ${ }^{9}$ Potential biological underpinnings of

20 insomnia with short sleep duration have also been explored, and this insomnia phenotype has

21 been found to be associated with multiple markers of hyperarousal and cardiometabolic risk. ${ }^{11}$ This has led some to describe insomnia with objective short sleep duration as the most "biologically severe" form of insomnia. ${ }^{11}$

24 It seems clear that insomnia, short sleep duration, and in particular their combination, predict prevalence and/or onset of mental health symptoms and associated biomarkers. However, the 
1 factors which precipitate an illness are not necessarily the same as those which maintain it, ${ }^{12-}$

$2{ }^{13}$ and the extent to which these dimensions of sleep disturbance are associated with mental

3 health symptom trajectory after onset is currently unclear. Clarifying this association would

4 have particular clinical utility as sleep disturbance is common among those with mental

5 disorders yet remains undertreated. ${ }^{14}$

6 To date, investigation of the impact of insomnia and/or short sleep duration on mental health

7 symptom trajectory has been limited by a) considering only a single dimension of sleep

8 disturbance at a time, b) potential confounders, and c) issues of measurement. ${ }^{15-19}$

9 A number of studies have found a prospective link between insomnia and chronicity of

10 mental health symptoms. ${ }^{15-17}$ However, these have not considered sleep duration, and not

11 controlled for important potential confounders such as past mental illness, physical activity,

12 low social support, stressful life events, or obesity.

13 Another study by Glozier et al. found that short sleep duration was associated with persistent

14 psychological distress in young adults $12-18$ months after baseline. ${ }^{18}$ While this study

15 controlled for multiple important confounders, only one dimension of sleep disturbance was

16 considered.

17 Finally, one study has considered multiple dimensions of sleep disturbance and trajectories of

18 mental health symptoms, while also controlling for important confounders, but this study also

19 has limitations. ${ }^{19}$ Van Mill et al. found that both short and long sleep duration, but not

20 insomnia, were associated with a chronic course of depression and anxiety, and found no

21 interaction between sleep duration and insomnia. ${ }^{19}$ They conclude that short and long sleep

22 duration - but not insomnia - predict a chronic course of mental illness, independent of

23 disorder symptom severity, and recommend routine assessment of sleep duration to identify

24 subjects at risk of a chronic course. However, the measure and cut-point used to define

25 insomnia were validated in a specific sample (postmenopausal women, mean 67.71 years of 
1 age), and the cut-point used yielded limited sensitivity (53\%) and specificity (67\%). ${ }^{20}$

2 Therefore, a significant proportion of the sample may have been misclassified with regard to

3 insomnia status, making it difficult to interpret the observed lack of associations with

4 insomnia.

5 The limitations described mean the extent to which insomnia, sleep duration, and their

6 combination predict a chronic trajectory of mental ill-health is currently unclear. If both

7 dimensions of sleep disturbance predict a chronic trajectory, this would suggest that it may be

8 necessary to identify and specifically target each, where they are occurring, in order to

9 maximise the odds of a positive long-term outcome.

\section{$10 \quad 1.1$ Aims of the Study}

11 The current study aimed to test three primary hypotheses:

1. Insomnia and suboptimal sleep duration (short and/or long duration) will have a prospective association with chronicity of mental health symptoms.

2. The combination of insomnia symptoms and short sleep duration will be more strongly associated with chronicity of mental health symptoms than either dimension of sleep disturbance in isolation.

3. These associations will be robust to adjustment for potential sociodemographic,

\section{Methods}

\section{1 \\ 2.1 Data}

22 Data were obtained through the ongoing Household, Income and Labour Dynamics in 23 Australia (HILDA) panel survey. ${ }^{21}$ The survey was approved by the Human Research Ethics

24 Committee of the University of Melbourne. Commencing in 2001, this survey annually 25 collects information related to labour market dynamics, family life, and personal well-being. 
1 It employed a multi-stage sampling approach to obtain a nationally representative sample of

2 households. Selected households were sent information via mail, and later invited to

3 participate directly by interviewers. At wave 1 (2001-02), 19,914 individuals participated (a

4 response rate of 66\%). In 2011-12 a further 5,451 persons were added to the cohort (a

5 response rate of $69 \%$ ).

6 Figure 1 summarises the selection of participants into the study. Baseline data for this study

7 were collected in 2013-14 (wave 13), the first wave in which sleep was assessed. 17,501

8 individuals participated at this time point. Follow up data were obtained after one year (2014-

9 15; wave 14) and after two years (2015-16; wave 15). Of those who participated in the survey

10 at baseline, $15,394(88.0 \%)$ also participated in the survey at both follow-up timepoints. Of

11 these, 12,525 participants (81.4\%) had available mental health data at each timepoint. 2,598

$12(20.7 \%)$ of those with full mental health data met criteria for elevated mental health symptoms (described in section 2.2) at baseline and were included in the study.

14 Although adolescents experience distinct changes in sleep timing and duration, ${ }^{22}$ they were

15 retained in the sample as sleep disturbance has been associated prospectively with mental

16 illness among this age group. ${ }^{23}$

17 Interviews were generally conducted at participant household addresses, with $<10 \%$ conducted via phone. The HILDA survey consists of two sections. Each member of a participating household over the age of 15 was invited to complete the first section with an 20 interviewer, and the second section alone (without interviewer administration). The second 21 section, referred to as the 'self-completion questionnaire' was comprised of attitudinal and other questions which respondents may be more comfortable answering privately. After this section was completed, it was either left with the interviewer, collected at a later date, or returned by mail. The average time between completion of the self-completion questionnaire at baseline and one-year follow-up was 11.84 months (SD 1.14; $4.8 \%$ missing $\geq$ one date). 
1 The average time in between completion of the self-completion questionnaire at one-year and

2 two-year follow up was 11.91 months (SD 1.03; $4.7 \%$ missing $\geq$ one date).

\subsection{Mental illness}

Mental illness outcome. The five-item Mental Health Inventory (MHI-5) is a short questionnaire that can be used to detect patients with anxiety and depression in primary care. ${ }^{24}$ The measure demonstrates good internal consistency reliability (Cronbach's $\alpha=$ 0.84) ${ }^{25}$ Cuijpers et al. ${ }^{26}$ reported that a cut-point of 62 yielded acceptable ability to detect DSM-III-R depression and dysthymia (sensitivity 74\%, specificity 91\%) and anxiety disorders (GAD, panic, OCD; sensitivities 62-75\%, specificities 89-90\%). Participants were classified as experiencing elevated mental health symptoms (signifying a probable mental illness) at each timepoint for which their MHI-5 score was less-than-or-equal-to 62. Finally, a three-level outcome variable mental health symptom trajectory was created based on participants' mental health symptoms at one-year and two-year follow up. Not meeting the MHI-5 criteria at either follow-up time point was classed as recovery. Meeting the MHI-5 criteria at only one of the follow up time points was classed as intermittent mental health symptoms. Meeting the MHI-5 criteria at both follow-up time points was classed as chronic mental health symptoms.

\subsection{Insomnia symptoms and sleep duration}

Insomnia symptoms. Key DSM-V and ICD-10 criteria for insomnia include complaints of difficulty initiating or maintaining sleep at least 3 times per week ${ }^{27-28}$. Participants indicated how frequently over the past month they a) had trouble sleeping because they cannot get to sleep within 30 minutes, and b) had trouble sleeping because they wake up in the middle of the night or early morning. Potential responses included 'not during the past month', 'less than once a week', 'once or twice a week', 'three or four times a week', and 'five or more times a week'. Experiencing either difficulty at least 3 nights per week was categorized as 
1 difficulty initiating or maintaining sleep. The fact that many individuals may experience

2 difficulty initiating or maintaining sleep but have no complaint ${ }^{27-28}$ was also taken into

3 account by incorporating subjective sleep quality into the operationalization of insomnia

4 symptoms. Participants rated their overall sleep quality during the past month as "very good",

5 "fairly good", "fairly bad", or "very bad". Responses of "fairly bad" or "very bad" were

6 classified as poor subjective sleep quality. Finally, a dichotomous composite variable,

7 insomnia symptoms, was created, with insomnia defined as reporting both difficulty initiating

8 or maintaining sleep and poor subjective sleep quality.

9 Sleep duration. Employed participants were asked how many hours of sleep they usually

10 obtain on work nights and non-work nights. Participants who were not in paid employment at

11 baseline were asked how many hours of sleep they usually obtain on weekday and weekend nights. All participants were also asked whether they typically nap either during the daytime or in the evening, and if so, for how long. For the employed, hours of sleep per week was then calculated by adding a) the product of workday sleep hours and number of days worked in primary job, b) the product of non-workday sleep hours and number of days not worked in primary job, and c) weekly nap duration. For the unemployed, hours of sleep per week was calculated based on usual weekday and weekend sleep hours weighted according to number of weekday (5) and weekend (2) nights, and weekly nap duration. Average sleep duration was calculated by dividing hours of sleep per week by seven. As the relationship between sleep

20 duration and mental health is non-linear, with both short and long sleep duration being associated with worse outcomes,${ }^{29}$ sleep duration was then converted to a four-category variable ( $<6 \mathrm{~h}, \geq 6$ to $<7 \mathrm{~h}, \geq 7$ to $<8 \mathrm{~h}, \geq 8 \mathrm{~h}$ ). These cut-offs were selected as typically sleep duration of $<6 \mathrm{~h}$ is categorized as short, ${ }^{5,10,19}$ the range for normal sleep duration varies

24 between 6-8 hours, ${ }^{5,10}$ and splitting within this range allows for possible differences in 
1 strength of associations. For analyses $\geq 7$ to $<8$ h sleep duration was set as the reference

2 group, as this group had the lowest prevalence of chronic mental health symptoms.

\section{$3 \quad 2.4$ Potential covariates}

4 The current study accounted for a range of factors that are associated with sleep disturbance

5 and/or mental health symptoms. All potential covariates are from baseline unless otherwise

6 stated. Variable definitions/categorizations were based on previous research, ${ }^{30}$ and greater

7 detail is provided in Appendix, Table A.1.

9 Demographics and socioeconomic status. Education was classified as high (masters or

10 doctorate, graduate diploma, graduate certificate, bachelor or honours), intermediate

11 (advanced diploma, diploma, Certificate III or IV, completed high school), and low (not completed high school). Dichotomous variables indicated whether respondents were partnered (married or in a de facto relationship), employed, of Aboriginal or Torres Strait Islander background (assumed no if not confirmed), or spoke English as a second language

15 (assumed no if not confirmed). Equivalised household income was calculated according to 16 the 'modified Organization for Economic Cooperation and Development (OECD) scale', 31 with lower limits set to 0 (i.e. negative values were not permitted). Extreme scores at the upper-end of the distribution were top-coded, with upper limits set to the seventy-fifth percentile plus 1.5 times the interquartile range. Low social support was assessed with a 10-

20 item measure described in Milner et al..$^{32}$ Items require respondents to report level of 21 agreement with statements such as "I have no one to lean on in times of trouble" on an 8point Likert scale. The measure demonstrates good internal consistency reliability

23 (Cronbach's $\alpha=0.84$ ). ${ }^{31}$ Low social support was defined as average scores below 4.69, as

24 such scores have been found to be associated with poorer mental health. ${ }^{31}$ Stressful life events were based on endorsing the occurrence of at least one of a number of stressful life 
1 events (e.g. death of a family member, loss of relationship, loss of employment) in the two

2 years prior to $\mathrm{T} 3$ (reported at $\mathrm{T} 2 \& \mathrm{~T} 3) .{ }^{33}$

3 Physical health and related behaviours: Binary variables reflected physical health

4 characteristics and behaviours as follows: Pain based on ratings of 'severe' or 'very severe'

5 on a 6-point scale, ${ }^{34}$ drinking behaviour based on drinking alcohol 5 days per week or more, ${ }^{35}$

6 smoking behaviour based on smoking daily, ${ }^{36}$ obesity based on a BMI of 30 or higher, ${ }^{37}$

7 chronic illness based on self-reported diagnoses of type I or II diabetes, heart disease or

8 circulatory disease, hypertension, asthma, cancer, arthritis or osteoporosis, or other serious

9 illness, with the exception of mental health diagnoses ${ }^{38}$; potential undiagnosed sleep apnea

10 based on self-reported trouble sleeping due to coughing or snoring at least 3 nights per

11 week, ${ }^{39}$ and physical activity based on moderate-to-vigorous physical activity more than 3

12 times per week. ${ }^{40}$

13 Mental illness and treatment: The presence of a previous episode of mental illness (of which

14 the insomnia symptoms could be a residual symptom) was defined as meeting the above

15 MHI-5 criteria at any of waves 1-12 after initially joining the study (i.e. up to twelve years

16 previously). Self-reported sleep and other psychiatric medication use was also obtained, and

17 the MHI-5 was included as a continuous measure of mental health.

$18 \quad 2.5$ Statistical analyses

19 All analyses were conducted using SPSS version 25 (IBM). Descriptive statistics were

20 obtained for the final sample, with differences between groups defined by mental health

21 symptom trajectory assessed via t-tests and chi-squared tests. Univariate associations between

22 mental health symptom trajectory and each of the predictors and covariates, and associations

23 between sleep variables (insomnia symptoms and sleep duration) and covariates were

24 assessed via logistic regression. 
1 Associations between sleep variables and mental health symptom trajectory were then

2 adjusted for covariates that were found to be associated with both the outcome measure and

3 at least one of the sleep variables. For adjusted models, multiple imputation was used to

4 impute missing values ${ }^{41}$ with the Markov chain Monte Carlo method, under the assumption

5 that data were missing at random (MAR), using all independent variables and two-way

6 interaction terms as predictors. Estimates from 30 imputed samples were pooled for

7 analysis. $^{42}$

8 The analysis was hierarchical, with mental health symptom trajectory first regressed on

9 baseline insomnia symptoms or sleep duration (in separate analyses), adjusting for age and

10 sex. In subsequent steps, other covariates were added to the model in blocks ordered from

11 those expected to have a lesser attenuating effect on the coefficient for sleep variables, to

12 those expected to have the greatest attenuating effect or found to be most strongly associated

13 with mental illness outcome. After the first step, other sociodemographic covariates were

14 added to the model, followed by physical health covariates, then mental health covariates. For

15 each model, linearity of the relationships between logits and predictors was assessed using

16 the Box-Tidwell procedure. For continuous covariates which were nonlinear with the logit,

17 two polynomial terms were created by respectively squaring and cubing the continuous

18 variable. ${ }^{43}$ These polynomial terms were entered with the associated continuous variable (e.g.

19 age-squared and age-cubed entered with age). Quadratic terms were included at each step of

20 the model at which either it or the cubic term was significant, while a cubic term was only

21 included if it was significant.

22 In another model both sleep variables were added to fully-adjusted models to determine

23 whether each had an independent effect on mental health symptom trajectory.

24 In a separate model, interactions between insomnia symptoms and sleep duration, adjusted

25 for covariates, were assessed. Interactions between insomnia symptoms and each of the 
1 covariates were also included in this model to take into account potential variation in

2 coefficient slopes based on factors other than the potential moderator of interest, and the

3 sample was large enough for inclusion of these additional terms to be feasible. Interactions

4 between sleep variables and age group ( $<25$ years, 25-44 years, 45-64 years, $65+$ years $){ }^{30}$

5 sex, and past mental health were also assessed in this manner.

6 The treatment of multiple-level categorical variables in regression analyses would not allow

7 all possible categories to be compared. Therefore, as in previous studies, ${ }^{10}$ we also created a

8 categorical variable with 6 groups defined by combinations of insomnia symptoms and sleep

9 duration (categories in Table 4). For this analysis, sleep duration of $<6 \mathrm{~h}$ or $\geq 6$ to $<7 \mathrm{~h}$ were

10 defined as "short sleep duration". The initial analysis was then repeated using this "joint sleep

11 variable", with the combination of no insomnia symptoms and $\geq 7$ to $<8 \mathrm{~h}$ sleep duration as

12 the reference group.

13 All regressions were re-run with original (non-imputed) data, to assess whether associations

14 of insomnia symptoms or sleep duration with mental illness outcome were sensitive to data

15 imputation. Any differences between analyses using imputed and original (non-imputed) data

16 are reported in the results.

\section{3. Results}

19 For those who had baseline mental health data, missing mental health data at one or two-year

20 follow-up was negatively associated with elevated mental health symptoms at baseline, but

21 not with insomnia symptoms or sleep duration. Missing data comprised $0-4.8 \%$ of values per

22 variable, with the exception of psychiatric medication (8.1\% missing), past mental illness

23 (9.1\%), and chronic illness (15.7\%). See Appendix Table A.2 for missing data proportions

24 and characteristics of original and imputed data sets. 


\section{$1 \quad 3.1$ Descriptive statistics}

2 Participants in the final sample ranged from 15 to 94 years of age $(\mathrm{M}=44.35, \mathrm{SD}=17.86)$.

3 This included 125 individuals < 18 years of age. At two-year follow-up, a total of 673

4 participants (25.9\% of the sample) were assessed as meeting criteria for recovery, 711

$5(27.4 \%)$ as experiencing intermittent mental health symptoms, and 1,214 (46.7\%) as

6 experiencing chronic mental health symptoms. A total of 1,056 (40.6\%) participants were

7 assessed as experiencing insomnia symptoms at baseline. Characteristics of the sample are

8 found in Table 1, stratified by mental health symptom trajectory. See Table 2 for associations

9 between insomnia symptoms and sleep duration.

10 In univariate logistic regression analyses the majority of independent variables $(n=19 / 23)$

11 were associated with mental health symptom trajectory. All covariates associated with mental

12 health symptom trajectory were also associated with insomnia symptoms and/or sleep

13 duration. See Appendix Tables A.3-A.5 for associations of mental health symptom trajectory

14 and sleep variables with other predictors/covariates.

\section{$15 \quad 3.2$ Insomnia symptoms}

16 Associations of sleep variables with mental health symptom trajectory are outlined in Table

17 3. Adjusted for age and sex, insomnia symptoms were associated with intermittent mental

18 health symptoms (odds ratio (OR) $1.43,95 \%$ CI $1.15-1.80$ ) and chronic mental health

19 symptoms (OR 2.16, 95\% CI 1.77 - 2.65). After adjustment for physical health covariates

20 insomnia symptoms were no longer associated with intermittent mental health symptoms (OR

21 1.23, 95\% CI $0.97-1.56)$. After mental health covariates were accounted for, insomnia symptoms were still associated with chronic mental health symptoms (OR 1.40, 95\% CI 1.11

23 - 1.76). When further adjusted for sleep duration, insomnia symptoms remained a significant

24 predictor of chronic mental health symptom (OR 1.34, 95\% CI $1.05-1.72$ ). 
1 As those with "no insomnia symptoms" included those with difficulties initiating and

2 maintaining sleep (DIMS) but normal sleep quality, we considered the possibility that this

3 subgroup may differ from the remainder of the "no insomnia symptoms" group i.e. those with

4 no DIMS and normal sleep quality. However, post-hoc analyses indicated that difficulties

5 initiating or maintaining sleep in the absence of poor sleep quality was not associated with

6 greater adjusted odds of intermittent (OR 1.03, 95\%CI $0.68-1.57$ ) or chronic (OR 1.28, 95\%

7 CI 0.86 - 1.91) mental health symptom trajectories, compared to those with no DIMS and

8 normal sleep quality.

\section{$9 \quad 3.3$ Sleep duration}

10 Sleep duration was associated with chronic mental health symptoms after adjustment for

11 sociodemographics, physical health, and mental health. Sleeping < 6h (OR 1.48, 95\% CI 1.11

$12-1.98), \geq 6$ to $<7$ h $($ OR $1.65,95 \%$ CI $1.23-2.22)$ and $>8$ h (OR $1.34,95 \%$ CI $0.99-1.81)$

13 per night were associated with greater likelihood of chronic mental health symptoms at 2-

14 year follow-up compared to sleeping $\geq 7$ to $<8 \mathrm{~h}$ per night, though statistical significance

15 was not reached for $>8 \mathrm{~h}$.

16 After adjustment for insomnia symptoms, the trend remained for sleep duration to be

17 association with chronic mental health symptoms. Sleeping < 6 h (OR 1.32 95\% CI $0.97-$

$181.80, \mathrm{p}=.077), \geq 6$ to $<7 \mathrm{~h}(\mathrm{OR} 1.59,95 \%$ CI $1.18-2.14, \mathrm{p}=.002)$, and $\geq 8 \mathrm{~h}$ per night (OR

$191.36,95 \%$ CI $1.00-1.84, \mathrm{p}=.047)$ continued to be associated with chronic mental health

20 symptoms. The pattern was similar using non-imputed data, though sleeping $<6 \mathrm{~h}(\mathrm{OR} 1.50$,

$2195 \%$ CI $1.04-2.16)$ but not $\geq 8$ h (OR 1.42, 95\% CI $0.98-2.05, \mathrm{p}=.064)$ reached statistical 22 significance.

23 In a sensitivity analysis, when $<6$ h sleep duration was further broken down into $\leq 5 \mathrm{~h}(\mathrm{n}=$

24367 in original data $)$ and $>5$ to $<6 \mathrm{~h}(\mathrm{n}=362$ in original data $)$, sleeping $\leq 5 \mathrm{~h}(\mathrm{OR} 1.90,95 \%$ 
1 CI $1.29-2.79)$ but not $>5$ to $<6 \mathrm{~h}(\mathrm{OR} 1.25,95 \%$ CI $0.89-1.75)$ was associated with

2 chronic mental health symptoms after full covariate adjustment.

\section{$4 \quad 3.4$ Interactions}

5 There was no significant interaction between insomnia symptoms and sleep duration.

6 However, the analysis using the 'joint sleep variable' defined by combinations of insomnia

7 symptoms and sleep duration suggested that insomnia symptoms had a greater independent

8 association with chronic mental health symptoms when combined with $<7$ h per night sleep

9 duration (Table 4).

10 There were no significant interactions between sleep variables and sex.

11 There was no significant interaction between age group and insomnia symptoms. There was a significant interaction between age group and sleep duration. Sleeping $<6 \mathrm{~h}$ per night was more strongly associated with chronic mental health symptoms for those aged 45-64 years than those aged $<25$ years (OR 2.51, 95\% CI $1.04-6.09, \mathrm{p}=.041)$. There was also a trend for sleeping $<6 \mathrm{~h}$ per night to be more associated with chronic mental health symptoms for those aged 25-44 years than those aged $<25$ years (OR 2.32, 95\% CI $0.93-5.80, p=.072)$, which was significant using non-imputed data. There was also a significant interaction between insomnia symptoms and past mental illness (OR 2.49, 95\% CI $1.17-5.33, \mathrm{p}=.019)$. For those with past mental illness, insomnia symptoms, fully-adjusted, were associated with greater risk of chronic mental health symptoms at two-year follow-up (OR 1.56, 95\% CI 1.21 - 2.01), while for those without past mental illness insomnia symptoms were not associated with increased risk of chronic mental health symptoms (OR $0.7395 \%$ CI 0.32 - 1.68). This interaction was not significant using non-imputed data (OR 2.26, 95\% CI $0.98-5.22, \mathrm{p}=.055)$, but the pattern of a significant association for those with a prior history of mental illness and lack of association for those 
1 without such a history remained. There was no interaction between sleep duration and past

2 mental illness.

\section{4. Discussion}

4 To our knowledge, this is the first study to show that both insomnia and sleep duration, alone

5 and in combination, are associated with the trajectory of elevated mental health symptoms

6 after adjustment for a wide range of potential confounders, in a sample aged across the adult

7 lifespan. Insomnia symptoms were more predictive of a chronic trajectory for those with a

8 history of probable mental illness. The combination of insomnia symptoms with short sleep

9 duration $(<7 \mathrm{~h}$ per night) was more predictive of chronic mental health symptoms than either

10 dimension of sleep disturbance alone.

11 The findings related to insomnia symptoms were partly consistent with most previous

12 research. They are in line with the results of previous studies which suggest an association

13 between insomnia and mental illness chronicity. ${ }^{15-17}$ The current results build on these

14 findings by adjusting for a range of potential confounders not previously considered.

15 There is also a lack of previous research assessing potential interaction between sleep

16 disturbance and mental health history. We observed that insomnia symptoms were more

17 predictive of chronic mental health symptoms for those with a history of likely mental illness.

18 It is possible that those with a history of mental illness are more likely to be experiencing

19 chronic (rather than acute) insomnia, and that this stable insomnia is associated with worse

20 mental health prognosis. However, the hypothesis was untestable here as no measure of

21 insomnia symptom chronicity was available, and the possibility warrants future exploration.

22 Our findings related to sleep duration are also consistent with previous research. Large-scale

23 studies have found that after adjustment for potentially confounding variables, shorter sleep

24 duration was associated with increased risk of persistently elevated psychological distress 12-

2518 months later, ${ }^{18}$ and 2 -year chronic course trajectory of depression/anxiety. ${ }^{19}$ We also 
1 found that short and long sleep duration were associated with a chronic course trajectory of

2 mental health symptoms, after adjusting for a wider range of covariates than in previous

3 research (though long sleep duration did not reach statistical significance).

$4 \quad$ Perhaps the primary finding of the current study was that the combination of insomnia

5 symptoms and short sleep duration were more predictive of a chronic course of mental health

6 symptoms than either insomnia or short sleep duration alone. This extends the findings of

7 previous studies. ${ }^{7,9}$ Furihata et al. ${ }^{7}$ observed an "additive" effect of sleep disturbance

8 dimensions on depression incidence in a population of older women (mean age 80.1 years). ${ }^{7}$

9 Fernandez-Mendoza et al. ${ }^{9}$ found that insomnia with short sleep duration is associated with

10 incident depression independent of coping resources and other covariates. The current study

11 demonstrates a similar "combined" effect of insomnia symptoms and short sleep duration, but

12 in this case shows that a) this effect is also associated with trajectory (as opposed to

13 incidence) of mental health symptoms, b) this effect is robust to adjustment for a wider range

14 of confounders than those included previous studies.

15 It should be noted that one previous study adjusting for a range of confounders suggested that

16 insomnia does not contribute to the impact of sleep disturbance on chronicity of common

17 mental disorders. ${ }^{19}$ However, this may be explained by differences in measurement. Rather

18 than using diagnostic criteria, insomnia in this earlier study was determined using a measure

19 which has previously been found to have limited sensitivity (53\%) and specificity (67\%)

20 when used to detect insomnia, and was validated in an age-and-sex-restricted sample of

21 postmenopausal women. ${ }^{20}$ Resultant misclassification may have prevented the identification

22 of a relationship between insomnia and illness chronicity. Furthermore, the finding in the

23 current study that the combination of insomnia and short sleep duration is more strongly

24 associated with mental health chronicity than either sleep dimension in isolation is supported

25 by growing evidence that insomnia with objective short sleep duration is the most 
1 "biologically severe" form of the disease, as evidenced by associations with multiple

2 physiological markers of hyperarousal. ${ }^{11}$

3 It is important to consider that in theory, the sleep disturbance which is associated with

4 chronicity of mental ill-health may simply be a residual symptom of prior mental illness, or

5 indicative of more severe mental health symptoms at baseline, rather than a

6 causal/maintaining factor. However, these factors (past mental health and baseline mental

7 health symptoms) were controlled for in the current analyses. Furthermore, it is also possible

8 that the attenuation of effects following covariate adjustment to some degree represents over

9 adjustment. ${ }^{44}$ For example, controlling for an intermediate variable on a causal path from

10 sleep disturbance to mental health symptom chronicity (e.g. continuous mental health at

11 baseline, possibly a function of sleep disturbance) may bias the effect of sleep disturbance

12 towards the null.

13 Therefore, the significant association of insomnia symptoms and sleep duration with mental

14 health symptom trajectory, even after adjustment for multiple confounders, suggests that

15 maximising the probability of mental illness recovery may require targeting sleep disturbance

16 when present, regardless of other illness-maintaining factors, notably including the baseline

17 severity of mental health symptoms and past mental illness. It is also noteworthy that

18 associations of sleep disturbance with mental health symptom chronicity appeared strongest

19 for those with insomnia symptoms and short sleep duration combined as opposed to either in

20 isolation. Combined with evidence that insomnia with objective short sleep duration is more

21 biologically severe than insomnia with normal sleep duration, ${ }^{11}$ this suggests intervention is particularly indicated for those presenting with this phenotype. 


\section{$1 \quad$ 4.1 Limitations \& Future Work}

2 The strength of the above recommendations is tempered by several limitations in the present

3 study. It is possible that had complete diagnostic criteria been used to classify probable

4 mental illness (rather than a cut-point on a mental health scale), sleep disturbance may have

5 been more or less strongly associated with chronicity. The measure we used shows good

6 ability to discriminate between cases and non-cases of depression and anxiety, ${ }^{26}$ and excellent

7 specificity in particular means nearly the whole sample might be expected to meet criteria for

8 depression and/or anxiety. However, using diagnostic criteria would also make it possible to

9 determine whether sleep disturbance is predictive of illness chronicity for particular

10 diagnoses. The temporal "coverage" of the measures was also a limitation, as illness during

11 the 1-year period between each timepoint was not assessed. This means the true trajectory of

12 illness was not assessable. However, those who were ill at both follow-up timepoints might

13 be expected to be doing worse on average than those who were not, and so the measure is

14 likely at least a good proxy for chronicity. There were also limitations in the measurement of

15 covariates. For example, the measure of alcohol consumption accounted for frequency but not

16 quantity of consumption, and the measure of unemployment did not distinguish between

17 unemployment due to retirement versus inability to find work.

18 Another limitation is the reliance on self-report and potential associated inaccuracies. This is

19 especially relevant for our population, as those with mental illness are often not individually

20 accurate in estimating their habitual sleep duration. ${ }^{45}$ Further assessment of the relationship

21 between sleep disturbance and mental health symptom trajectories using objective measures

22 of sleep (and adjusting for potential confounders) is therefore warranted.

23 Finally, the results of the current study suggest that it may be especially important to address

24 insomnia when combined with short sleep duration, but do not indicate how. It is beyond the

25 scope of this paper to thoroughly explore this question. However, one notable hypothesis is 
1 that patients with insomnia and short sleep duration, who display higher physiological arousal

2 and poorer response to cognitive-behavioural interventions for insomnia (CBT-I), may

3 benefit from pharmacotherapy or combined therapy (CBT-I + medication). ${ }^{46}$ In any case, the

4 current results indicate that research confirming effective interventions for sleep disturbance

5 and its phenotypes in psychiatric populations may aid in optimising mental health symptom

6 trajectories.

\section{$7 \quad 4.2$ Conclusion}

8 Notwithstanding acknowledged limitations, the results of the current study suggest that

9 insomnia and short sleep duration are not simply symptoms of physical or mental illness, but

10 predict a chronic trajectory of elevated mental health symptoms. This speaks to a need for

11 those with mental health symptoms and their clinicians to identify and address sleep

12 disturbance (i.e. insomnia and/or short sleep duration) when present, regardless of other

13 illness-maintaining factors. This may be particularly important for those presenting with the

14 combination of insomnia and short sleep duration.

16 Acknowledgements: This paper uses unit record data from the Household, Income and

17 Labour Dynamics in Australia (HILDA) Survey. The HILDA Project was initiated and is

18 funded by the Australian Government Department of Social Services (DSS) and is

19 managed by the Melbourne Institute of Applied Economic and Social Research

20 (Melbourne Institute). The findings and views reported in this paper, however, are those of

21 the authors and should not be attributed to either DSS or the Melbourne Institute. NG serves on the advisory board for Lundbeck, receives honoraria from Lundbeck and MSD, and is supported by grants from NHMRC of Australia. TL is supported by the Academy of

24 Finland (Grants \#287488 and \#319200).

25 Funding: This research did not receive any specific grant from funding agencies in the 26 public, commercial, or not-for-profit sectors. 


\section{References}

1. Jansson-Fröjmark M, Lindblom K. A bidirectional relationship between anxiety and depression, and insomnia? A prospective study in the general population. $J$. Psychosomatic Res. 2008; 64(4): 443-449.

2. Riemann D. Insomnia and comorbid psychiatric disorders. Sleep Med. 2007; 8: S15-S20.

3. Baglioni C, Battagliese G, Feige B, et al. Insomnia as a predictor of depression: a metaanalytic evaluation of longitudinal epidemiological studies. J. Affect. Disord. 2011; 135(1): 10-19.

4. Batterham PJ, Glozier N, Christensen H. Sleep disturbance, personality and the onset of depression and anxiety: prospective cohort study. Aust. N. Z. J. Psychiatry. 2012; 46(11):1089-1098.

5. Zhai L, Zhang H, Zhang D. Sleep duration and depression among adults: A meta-analysis of prospective studies. Depression and anxiety. 2015; 32(9): 664-670.

6. Roberts RE, Duong HT. Is there an association between short sleep duration and adolescent anxiety disorders?. Sleep Med. 2017; 30: 82-87.

7. Furihata R, Hall MH, Stone KL, et al. An aggregate measure of sleep health is associated with prevalent and incident clinically significant depression symptoms among community-dwelling older women. Sleep. 2017; 40(3): zsw075.

8. Kalmbach DA, Pillai V, Arnedt JT, Drake CL. DSM-5 insomnia and short sleep: comorbidity landscape and racial disparities. Sleep. 2016; 39(12):2101-2111.

9. Fernandez-Mendoza J, Shea S, Vgontzas AN, Calhoun SL, Liao D, \& Bixler EO. Insomnia and incident depression: role of objective sleep duration and natural history. J. Sleep Res. 2015; 24(4): 390-398.

10. Lallukka T, Sivertsen B, Kronholm E, Bin YS, Øverland S, Glozier N. Association of sleep duration and sleep quality with the physical, social, and emotional functioning among Australian adults. J. Sleep Health. 2018; 4(2): 194-200.

11. Fernandez-Mendoza J. The insomnia with short sleep duration phenotype: an update on its importance for health and prevention. Curr. Opin. Psychiatry. 2017; 30(1):56-63.

12. Stahl SM, Moore BA. (Eds.). (2013). Anxiety disorders: A guide for integrating psychopharmacology and psychotherapy. London: Routledge; 2013.

13. Alexander J, \& Treasure J. (Eds.). (2013). A collaborative approach to eating disorders. London: Routledge; 2013. 
14. Sunderajan P, Gaynes BN, Wisniewski SR, et al. Insomnia in patients with depression: a STAR* D report. CNS Spectr. 2010; 15(6):394-406.

15. Li S, Chan J, Lam J, Yu M, Wing Y. Can nocturnal sleep disturbances predict nonremission and relapse in patients with major depressive disorder? VA 5-year naturalistic longitudinal study. Sleep Med. 2013; 14:e48.

16. Pigeon WR, Hegel M, Unützer J, et al. Is insomnia a perpetuating factor for late-life depression in the IMPACT cohort? Sleep. 2008; 31(4):481-488.

17. Chan JWY, Lam SP, Li SX, et al. Eveningness and insomnia: independent risk factors of nonremission in major depressive disorder. Sleep. 2014; 37(5): 911-917.

18. Glozier N, Martiniuk A, Patton G, et al. Short Sleep Duration in Prevalent and Persistent Psychological Distress in Young Adults: The DRIVE Study. Sleep. 2010; 33(9): 1139-1145.

19. van Mill J, Vogelzangs N, van Someren E, Hoogendijk W, Penninx B. Sleep duration, but not insomnia, predicts the 2-year course of depressive and anxiety disorders. J. Clin. Psychiatry. 2014; 75(2):119-126.

20. Levine DW, Lewis MA, Bowen DJ, et al. Reliability and validity of Women's Health Initiative Insomnia Rating Scale. Psychol. Assess. 2003; 15(2):137.

21. Watson N, Wooden MP. The HILDA Survey: a case study in the design and development of a successful household panel survey. Longit. Life Course Stud. 2012; 3(3):369-381.

22. Carskadon MA, Acebo C, Jenni OG. Regulation of adolescent sleep: implications for behavior. Ann. N. Y. Acad. Sci. 2004; 1021(1): 276-291.

23. Lovato N, Gradisar M. A meta-analysis and model of the relationship between sleep and depression in adolescents: recommendations for future research and clinical practice. Sleep Med. Rev. 2014; 18(6):521-529.

24. Berwick DM, Murphy JM, Goldman PA, Ware Jr JE, Barsky AJ, Weinstein MC. Performance of a five-item mental health screening test. Med. Care. 1991; 29(2):169176.

25. McCabe CJ, Thomas KJ, Brazier JE, Coleman P. Measuring the mental health status of a population: A comparison of the GHQ-12 and the SF-36 (MHI-5). $B r J$. Psychiatry. 1996; 169(4):517-521.

26. Cuijpers P, Smits N, Donker T, Ten Have M, de Graaf R. Screening for mood and anxiety disorders with the five-item, the three-item, and the two-item Mental Health Inventory. Psychiatry Res. 2009; 168(3): 250-255. 
1 27. American Psychiatric Association. Diagnostic and statistical manual of mental disorders (fifth edition). Washington: American Psychiatric Association Publishing; 2013.

28. World Health Organization. The ICD-10 classification of mental and behavioural disorders: clinical descriptions and diagnostic guidelines. Geneva: World Health Organization; 1992.

29. Gadie A, Shafto M, Leng Y, Kievit RA. How are age-related difference in sleep quality associated with health outcomes? An epidemiological investigation in a UK cohort of 2406 adults. bioRxiv. 2017; 060145.

30. Biddle DJ, Kelly PJ, Hermens DF, Glozier N. The association of insomnia with future mental illness. Is it just residual symptoms? J. Sleep Health. In press. https://doi.org/10.1016/j.sleh.2018.05.008

31. Hagenaars AJ, De Vos K, Asghar Zaidi M. Poverty statistics in the late 1980s: Research based on micro-data. Eurostat. 1994.

32. Milner A, Krnjacki L, LaMontagne A. Age and gender differences in the influence of social support on mental health: a longitudinal fixed-effects analysis using 13 annual waves of the HILDA cohort. Public Health. 2016; 140:172-178.

33. Musliner KL, Seifuddin F, Judy JA, Pirooznia M, Goes FS, Zandi, PP. Polygenic risk, stressful life events and depressive symptoms in older adults: a polygenic score analysis. Psychol. Med. 2015; 45(8):1709-1720.

34. Schurer S, Shields MA, Jones AM. Socio-economic inequalities in bodily pain over the life cycle: longitudinal evidence from Australia, Britain and Germany. J. R. Stat. Soc. Ser. A. 2014; 177(4):783-806.

35. Kavanagh A, Krnjacki ML. Accessibility to alcohol outlets and alcohol consumption: Findings from VicLANES. Carlton, Australia: Victorian Health Promotion Foundation [VicHealth]; 2011.

36. John U, Meyer C, Rumpf, HJ, Hapke U. Smoking, nicotine dependence and psychiatric comorbidity - a population-based study including smoking cessation after three years. Drug Alcohol Depend. 2004; 76(3):287-295.

37. Williams EP, Mesidor M, Winters K, Dubbert PM, Wyatt SB. Overweight and obesity: prevalence, consequences, and causes of a growing public health problem. Curr. Obes. Rep. 2015; 4(3):363-370.

38. Chesher NJ, Bousman CA, Gale M, et al. Chronic illness histories of adults entering treatment for co-occurring substance abuse and other mental health disorders. Am.J. Addict. 2012; 21(1): 1-4. 
1 39. Nieto FJ, Young TB, Lind BK, et al. (2000). Association of sleep-disordered breathing, sleep apnea, and hypertension in a large community-based study. JAMA. 2000; 283(14):1829-1836.

40. Wen CP, Wai JP, Tsai MK, et al. Minimum amount of physical activity for reduced mortality and extended life expectancy: a prospective cohort study. Lancet. 2011; 378(9798):1244-1253.

41. Rubin DB. Multiple imputation for nonresponse in surveys (Vol. 81). Hoboken, NJ: John Wiley \& Sons; 2004.

42. Graham JW, Olchowski AE, Gilreath TD. How many imputations are really needed? Some practical clarifications of multiple imputation theory. Prev. Sci. 2007; 8(3):206213.

43. Osborne JW. Best practices in logistic regression. USA: Sage Publications; 2015.

44. Schisterman EF, Cole SR, Platt RW. Overadjustment bias and unnecessary adjustment in epidemiologic studies. Epidemiology. 2009; 20(4):488-495.

45. Biddle DJ, Robillard R, Hermens DF, Hickie IB, Glozier N. Accuracy of self-reported sleep parameters compared with actigraphy in young people with mental ill-health. $J$. Sleep Health. 2015; 1(3):214-220.

46. Bastien CH, Ellis JG, Grandner M. (2017). CBT-I and the short sleep duration insomnia phenotype: a comment on Bathgate, Edinger and Krystal. Ann. Transl. Med., 5(16): 335. 
Table 1. Characteristics of the sample as a whole and stratified by mental health symptom trajectory.

\begin{tabular}{|c|c|c|c|c|c|c|}
\hline & $\begin{array}{c}\text { Whole sample } \\
(\mathbf{n}=\mathbf{2 , 5 9 8})^{\dagger}\end{array}$ & $\begin{array}{c}\text { Recovery } \\
(n=673)\end{array}$ & $\begin{array}{c}\text { Intermittent } \\
(\mathbf{n}=711)\end{array}$ & $\begin{array}{c}\text { Chronic } \\
(\mathrm{n}=\mathbf{1 , 2 1 4})\end{array}$ & & \\
\hline & Mean (SD) & Mean (SD) & Mean (SD) & Mean (SD) & $\mathbf{F}$ & $\mathbf{p}$ \\
\hline Age & $44.35(17.86)$ & $42.7(17.5)$ & $42.5(18.0)$ & $46.4(17.8)$ & 14.63 & $<.001$ \\
\hline Household income (\$10k’s) & $9.62(6.68)$ & $10.72(6.96)$ & $9.71(6.52)$ & $8.96(6.55)$ & 15.31 & $<.001$ \\
\hline $\begin{array}{r}\text { Mental health (MHI-5 } \\
\text { continuous) }\end{array}$ & 48.03 (11.99) & $52.6(8.8)$ & $49.6(10.8)$ & $44.6(13.1)$ & 113.15 & $<.001$ \\
\hline Insomnia symptoms & $\begin{array}{c}\mathbf{n}(\%) \\
1056(40.6)\end{array}$ & $\begin{array}{c}\mathbf{n}(\%) \\
202(30.3)\end{array}$ & $\begin{array}{c}\mathbf{n}(\%) \\
270(38.6)\end{array}$ & $\begin{array}{c}\mathrm{n}(\%) \\
584(48.9)\end{array}$ & $\begin{array}{c}\chi^{2} \\
63.39\end{array}$ & $\begin{array}{c}\mathrm{p} \\
<.001\end{array}$ \\
\hline $\begin{array}{r}\text { Sleep duration } \\
<6 \mathrm{~h} \\
\geq 6 \text { to }<7 \mathrm{~h} \\
\geq 7 \text { to }<8 \mathrm{~h} \\
\geq 8\end{array}$ & $\begin{array}{l}729(28.3) \\
574(22.3) \\
708(27.5) \\
567(22.0)\end{array}$ & $\begin{array}{l}152(22.7) \\
146(21.8) \\
228(34.0) \\
144(21.5)\end{array}$ & $\begin{array}{l}192(27.3) \\
137(19.5) \\
216(30.7) \\
159(22.6)\end{array}$ & $\begin{array}{l}385(32.0) \\
291(24.2) \\
264(21.9) \\
264(21.9)\end{array}$ & 44.80 & $<.001$ \\
\hline Sex (female) & $1506(58.0)$ & $381(56.6)$ & $438(61.6)$ & $687(56.6)$ & 5.31 & .070 \\
\hline $\begin{array}{r}\text { Education (low) } \\
\text { Education (intermediate) } \\
\text { Education (high) }\end{array}$ & $\begin{array}{c}881(33.9) \\
1193(45.9) \\
524(20.2)\end{array}$ & $\begin{array}{l}200(29.7) \\
319(47.4) \\
154(22.9)\end{array}$ & $\begin{array}{l}258(36.3) \\
314(44.2) \\
139(19.5)\end{array}$ & $\begin{array}{l}423(34.8) \\
560(46.1) \\
231(19.0)\end{array}$ & 9.17 & .057 \\
\hline Partnered & $1470(56.6)$ & $404(60.0)$ & $400(56.3)$ & $666(54.9)$ & 4.75 & .093 \\
\hline Unemployed & $1221(47.0)$ & $244(36.3)$ & $323(45.4)$ & $654(53.9)$ & 54.91 & $<.001$ \\
\hline $\begin{array}{l}\text { ATSI background } \\
\text { English } 2^{\text {nd }} \text { language }\end{array}$ & $\begin{array}{c}84(3.2) \\
305(11.7)\end{array}$ & $\begin{array}{l}15(2.2) \\
66(9.8)\end{array}$ & $\begin{array}{c}22(3.1) \\
81(11.4)\end{array}$ & $\begin{array}{c}47(3.9) \\
158(13.0)\end{array}$ & $\begin{array}{l}3.79 \\
4.41\end{array}$ & $\begin{array}{l}.150 \\
.110\end{array}$ \\
\hline Low social support & $1350(53.2)$ & $243(37.0)$ & $359(51.8)$ & $748(63.1)$ & 116.32 & $<.001$ \\
\hline Stressful life events & $2103(81.5)$ & $542(81.1)$ & $590(83.3)$ & $971(80.6)$ & 2.31 & .315 \\
\hline Pain & $418(16.1)$ & $68(10.1)$ & $106(14.9)$ & $244(20.1)$ & 32.95 & $<.001$ \\
\hline Drinking $>4$ times/week & $322(12.5)$ & $86(12.9)$ & $81(11.5)$ & $155(12.9)$ & 0.90 & .638 \\
\hline Smoking daily & $522(20.2)$ & $107(16.0)$ & $143(20.3)$ & $272(22.5)$ & 11.32 & .003 \\
\hline Obesity & $718(29.0)$ & $154(23.8)$ & $208(30.8)$ & $356(31.0)$ & 11.89 & .003 \\
\hline Chronic illness & $1190(54.3)$ & $267(45.1)$ & $300(50.1)$ & $623(62.4)$ & 50.65 & $<.001$ \\
\hline Potential sleep apnea & $266(10.4)$ & $40(6.0)$ & $74(10.6)$ & $152(12.7)$ & 20.51 & $<.001$ \\
\hline Physical Activity > 3 times/week & $670(25.9)$ & $198(29.5)$ & $199(28.1)$ & $273(22.6)$ & 13.11 & .001 \\
\hline Past mental illness & $2082(88.1)$ & $441(75.6)$ & $554(87.0)$ & $1087(95.2)$ & 142.19 & $<.001$ \\
\hline Sleep medication $(1+$ per week $)$ & $474(18.5)$ & $43(6.8)$ & $86(13.1)$ & $280(25.4)$ & 107.56 & $<.001$ \\
\hline Psychiatric medication (regular) & $409(17.1)$ & $72(10.8)$ & $123(17.5)$ & $279(23.3)$ & 44.76 & $<.001$ \\
\hline
\end{tabular}

${ }^{\dagger}$ Reported n's and percentages of those in each mental health symptom trajectory category with listed characteristics are based on data pre-imputation. $\mathrm{N}$ missing values $(\%)$ : age $=0(.0)$, income $=0(.0)$, mental health (MHI-5 continuous) $=0$ $(.0)$, insomnia $=38(1.5)$, sleep duration $=20(0.8)$, sex $=0(.0)$, education $=0(.0)$, partnered $=0(.0)$, unemployed $=0$ $(.0)$, ATSI background $=0(.0)$, English $2^{\text {nd }}$ language $=0(.0)$, low social support $=62(2.4)$, stressful life events $=17$ (.7), pain $=5(.2)$, drinking $=21(.8)$, smoking $=18(.7)$, obesity $=125(4.8)$, chronic illness $=408(15.7)$, potential sleep apnea $=36(1.4)$, physical activity $=10(.4)$, past mental illness $=236(9.1)$, sleep medication $=29(1.1)$, other psychiatric medication $=210(8.1)$. 
Table 2. Association of insomnia symptoms with sleep duration.

\begin{tabular}{|c|c|c|c|c|c|}
\hline & & $\begin{array}{c}\text { No insomnia } \\
\text { symptoms } \\
(\mathrm{n}=\mathbf{1 , 4 9 6 / 2 , 5 4 1})^{\dagger} \\
\end{array}$ & $\begin{array}{c}\text { Insomnia } \\
\text { symptoms } \\
(\mathrm{n}=\mathbf{1 , 0 4 5 / 2 , 5 4 1})^{\dagger} \\
\end{array}$ & & \\
\hline Sleep duration & $\mathbf{N}$ & $\mathrm{n}(\%)^{\ddagger}$ & $\mathrm{n}(\%)^{\ddagger}$ & $\chi^{2}$ & $\mathbf{p}$ \\
\hline$<6 \mathrm{~h}$ & 716 & $207(13.8)$ & $509(48.7)$ & 411.48 & $<.001$ \\
\hline$\geq 6$ to $<7 \mathrm{~h}$ & 566 & $337(22.5)$ & $229(21.9)$ & & \\
\hline$\geq 7$ to $<8 \mathrm{~h}$ & 700 & $526(35.2)$ & $174(16.7)$ & & \\
\hline$\geq 8 \mathrm{~h}$ & 559 & $426(28.5)$ & $133(12.7)$ & & \\
\hline
\end{tabular}


Table 3. Adjusted associations of sleep variables with mental health symptom trajectory.

\begin{tabular}{|c|c|c|c|c|}
\hline & \multicolumn{2}{|c|}{$\begin{array}{c}\text { Intermittent mental health } \\
\text { symptoms } \\
(\mathrm{n}=711 / 2,598)\end{array}$} & \multicolumn{2}{|c|}{$\begin{array}{c}\text { Chronic mental health } \\
\text { symptoms } \\
(\mathrm{n}=\mathbf{1 , 2 1 4} / 2,598)\end{array}$} \\
\hline & $\operatorname{AOR}(95 \% \mathrm{CI})^{\dagger}$ & $\mathbf{p}$ & $\operatorname{AOR}(95 \% \mathrm{CI})^{\dagger}$ & $\mathbf{p}$ \\
\hline \multicolumn{5}{|l|}{ Insomnia symptoms (yes/no) } \\
\hline Age and sex adjusted & $1.43(1.15-1.80)$ & .002 & $2.16(1.77-2.65)$ & $<.001$ \\
\hline Sociodemographics adjusted $\mathrm{d}^{\ddagger}$ & $1.34(1.07-1.69)$ & .011 & $1.96(1.59-2.41)$ & $<.001$ \\
\hline Physical health adjusted $\S$ & $1.23(0.97-1.56)$ & .081 & $1.76(1.42-2.18)$ & $<.001$ \\
\hline Mental health adjusted & $1.11(0.87-1.41)$ & .406 & $1.40(1.11-1.76)$ & .004 \\
\hline \multicolumn{5}{|l|}{ Sleep duration (categories) } \\
\hline \multicolumn{5}{|l|}{ Age and sex adjusted } \\
\hline$<6 \mathrm{~h}$ & $1.33(1.00-1.77)$ & .052 & $2.06(1.59-2.68)$ & $<.001$ \\
\hline$\geq 6$ to $<7 \mathrm{~h}$ & $0.98(0.73-1.33)$ & .915 & $1.70(1.30-2.22)$ & $<.001$ \\
\hline$\geq 7$ to $<8 \mathrm{~h}$ & reference & & reference & \\
\hline$\geq 8 \mathrm{~h}$ & $1.15(0.86-1.55)$ & .335 & $1.57(1.19-2.05)$ & .001 \\
\hline \multicolumn{5}{|l|}{ Sociodemographics adjuste $\bar{d}^{\ddagger, \dagger \dagger}$} \\
\hline$<6 \mathrm{~h}$ & $1.19(0.89-1.59)$ & .250 & $1.72(1.31-2.26)$ & $<.001$ \\
\hline$\geq 6$ to $<7 \mathrm{~h}$ & $0.97(0.72-1.32)$ & .859 & $1.65(1.26-2.18)$ & $<.001$ \\
\hline$\geq 7$ to $<8 \mathrm{~h}$ & reference & & reference & \\
\hline$\geq 8 \mathrm{~h}$ & $1.08(0.80-1.46)$ & .602 & $1.43(1.08-1.90)$ & .013 \\
\hline \multicolumn{5}{|l|}{ Physical health adjusted $§$} \\
\hline$<6 \mathrm{~h}$ & $1.11(0.83-1.49)$ & .487 & $1.61(1.22-2.12)$ & $<.001$ \\
\hline$\geq 6$ to $<7 \mathrm{~h}$ & $0.95(0.70-1.29)$ & .755 & $1.63(1.23-2.15)$ & $<.001$ \\
\hline$\geq 7$ to $<8 \mathrm{~h}$ & reference & & reference & \\
\hline$\geq 8 \mathrm{~h}$ & $1.04(0.77-1.41)$ & .777 & $1.36(1.02-1.80)$ & .034 \\
\hline \multicolumn{5}{|l|}{ Mental health adjusted ${ }^{\mathbb{I}}$} \\
\hline$<6 \mathrm{~h}$ & $1.06(0.79-1.43)$ & 689 & $1.48(1.11-1.98)$ & .008 \\
\hline$\geq 6$ to $<7 \mathrm{~h}$ & $0.96(0.70-1.31)$ & .790 & $1.65(1.23-2.22)$ & $<.001$ \\
\hline$\geq 7$ to $<8 \mathrm{~h}$ & reference & & reference & \\
\hline$\geq 8 \mathrm{~h}$ & $1.03(0.76-1.40)$ & .835 & $1.34(0.99-1.81)$ & .056 \\
\hline
\end{tabular}

${ }^{\dagger}$ Based on multinomial logistic regressions, with recovery $(n=673 / 2,598)$ as reference group.

$\ddagger$ Adjusted for covariates from previous stages plus income, education, partnered, unemployment, English as 2nd language, low social support

$\S$ Adjusted for covariates from previous stages plus pain, physical activity, smoking, potential sleep apnea, obesity, chronic illness

" Adjusted for covariates from previous stages plus mental health continuous (MHI-5), past mental illness, sleep medication, psychiatric medication

${ }^{\dagger \dagger}$ Quadratic and cubic terms included for age 
Table 4. Associations of the joint sleep variable (insomnia symptoms, sleep duration) with mental health symptom trajectory.

\begin{tabular}{|c|c|c|c|c|c|}
\hline & \multirow[b]{2}{*}{$\mathbf{n}^{\dagger}$} & \multicolumn{2}{|c|}{$\begin{array}{c}\text { Intermittent mental health } \\
\text { symptoms } \\
(\mathbf{n}=711 / 2,598)\end{array}$} & \multicolumn{2}{|c|}{$\begin{array}{c}\text { Chronic mental health } \\
\text { symptoms } \\
(\mathrm{n}=\mathbf{1 , 2 1 4} / 2,598) \\
\end{array}$} \\
\hline & & $\operatorname{AOR}(95 \% \mathrm{CI})^{\ddagger}$ & $\mathbf{p}$ & $\operatorname{AOR}(95 \% \mathrm{CI})^{\ddagger}$ & $\mathbf{p}$ \\
\hline \multicolumn{6}{|l|}{ Age and sex adjusted } \\
\hline No insomnia symptoms, $<7 \mathrm{~h}$ & 544 & $1.01(0.74-1.38)$ & .946 & $1.44(1.08-1.92)$ & .012 \\
\hline Insomnia symptoms, $<7 \mathrm{~h}$ & 738 & $1.61(1.18-2.20)$ & .003 & $3.15(2.37-4.18)$ & $<.001$ \\
\hline No insomnia symptoms, $\geq 7$ to $<8 \mathrm{~h}$ & 526 & reference & & reference & \\
\hline Insomnia symptoms, $\geq 7$ to $<8 \mathrm{~h}$ & 174 & $1.53(0.97-2.40)$ & .067 & $1.81(1.18-2.78)$ & .006 \\
\hline No insomnia symptoms, $\geq 8 \mathrm{~h}$ & 426 & $1.29(0.92-1.79)$ & .136 & $1.63(1.19-2.22)$ & .002 \\
\hline Insomnia symptoms, $\geq 8 \mathrm{~h}$ & 133 & $1.26(0.73-2.20)$ & .409 & $2.70(1.67-4.37)$ & $<.001$ \\
\hline \multicolumn{6}{|l|}{ Adjusted for all covariates $\S$} \\
\hline No insomnia symptoms, $<7 \mathrm{~h}$ & & $0.91(0.62-1.33)$ & .613 & $1.34(0.92-1.94)$ & .126 \\
\hline Insomnia symptoms, $<7 \mathrm{~h}$ & & $1.28(0.86-1.91)$ & .217 & $2.35(1.60-3.45)$ & $<.001$ \\
\hline No insomnia symptoms, $\geq 7$ to $<8 \mathrm{~h}$ & & reference & & reference & \\
\hline Insomnia symptoms, $\geq 7$ to $<8 \mathrm{~h}$ & & $0.98(0.56-1.72)$ & .956 & $1.04(0.60-1.81)$ & .882 \\
\hline No insomnia symptoms, $\geq 8 \mathrm{~h}$ & & $1.15(0.75-1.75)$ & .518 & $1.46(0.96-2.22)$ & .077 \\
\hline Insomnia symptoms, $\geq 8 \mathrm{~h}$ & & $0.84(0.41-1.72)$ & .640 & $1.31(0.67-2.56)$ & .431 \\
\hline \multicolumn{6}{|l|}{${ }^{\dagger} \mathrm{n}$ 's based on original data. } \\
\hline
\end{tabular}

